\title{
DENSIDAD DE POBLACIÓN Y FERTILIZACIÓN NITROGENADA EN LA PRODUCCIÓN DE SEMILLA HÍBRIDA DE MAÍZ ${ }^{1}$
}

\author{
Francisco Cervantes-Ortíz ${ }^{2}$,Jorge Covarrubias-Prieto ${ }^{2}$, José Antonio Rangel-Lucio ${ }^{2}$, \\ Arturo Daniel Terrón-Ibarra ${ }^{3}$, Mariano Mendoza-Elos ${ }^{2}$, Ricardo Ernesto Preciado-Ortiz $^{3}$
}

\section{RESUMEN}

Densidad de población y fertilización nitrogenada en la producción de semilla híbrida de maíz. El presente estudio tuvo como objetivo determinar el efecto de la densidad de población y la fertilización nitrogenada en el rendimiento y calidad de semilla híbrida de maíz. El experimento se realizó en el Instituto de Nacional de Investigaciones Forestales, Agrícolas y Pecuarias-Campo Experimental Bajío (INIFAP-CEBAJ), en el ciclo P-V 2009. En la cruza CML 142 x CML 176, progenitor femenino del híbrido H-374C, se evaluaron tres densidades de población $(60,75$ y 90 mil plantas/ha) y tres niveles de nitrógeno $(150,250$ y $350 \mathrm{~kg} /$ ha). En la línea CML 176, progenitor femenino del híbrido H-469C, se evaluaron los niveles de población de 60, 80 y 100 mil plantas/ha y los mismos niveles de nitrógeno que en el híbrido anterior. Se midieron caracteres agronómicos, rendimiento, sus componentes y la calidad física y fisiológica de semilla. En la cruza CML 142 x CML 176, el nitrógeno no tuvo efecto significativo en los caracteres evaluados. La densidad de población afectó estadísticamente el rendimiento, la floración y el índice de prolificidad. En la línea CML 176, la densidad de población afectó significativamente el rendimiento, la floración y el índice de prolificidad, asimismo, modificó el diámetro y longitud de mazorca y el número de semillas por hilera. También los porcentajes de semilla útil, bola grande y el desecho, fueron modificados por este factor. El nitrógeno presentó diferencias estadísticas para el número de mazorcas por planta, el diámetro y la longitud. El peso de mil semillas, el peso volumétrico y el vigor a través de la prueba de envejecimiento acelerado no se modificaron por el efecto de los tratamientos en los dos genotipos evaluados.

Palabras clave: manejo agronómico, rendimiento de semilla, calidad física y fisiológica de semilla.

\begin{abstract}
Population density and application of nitrogen in the production of hybrid corn seed. The objective of this work was to evaluate the effect of population density and nitrogen fertilization on hybrid corn seed yield and quality. The experiment was conducted at the Instituto Nacional de Investigaciones Forestales, Agricolas y Pecuarias- Campo Experimental Bajío (INIFAP-CEBAJ) in the P-V cycle 2009. In the crosses CML 142 x CML 176, female parent of hybrid $\mathrm{H}-374 \mathrm{C}$, three population densities (60, 75 and 90000 plants/ ha) and three nitrogen levels $(150,250$ and $350 \mathrm{~kg} / \mathrm{ha})$ were evaluated. In line CML 176, female parent of hybrid H-469C, population levels of 60,80 and 100 thousand plants/ha and the same levels of nitrogen as the previous hybrid were evaluated. Agronomic traits, yield, components and the physical and physiological quality of seed were measured. In the crosses CML 142 x CML 176, the nitrogen had no significant effect on the characters evaluated. The population density significantly affected the yield, flowering and prolificacy index. In line CML 176, the population density also affected significantly the yield, flowering and prolificacy index and it also changed the diameter and length of pod and seed number per row. Other seed traits also modified by this factor were: useful percentage rates, large ball, and waste. The nitrogen presented statistical differences for the number of ears per plant, diameter and length of ear. The weight of a thousand seeds, the volumetric weight and vigor through the accelerated aging test were not modified by the effect of treatment in both evaluated genotypes.
\end{abstract}

Key words: agronomic management, seed yield, physical and physiological quality of seed.

\footnotetext{
Recibido: 9 de febrero, 2012. Aceptado: 1 de abril, 2013. Proyecto de investigación de tesis de maestría del primer autor. Instituto Nacional de Investigaciones Forestales, Agrícolas y Pecuarias, México.

2 Instituto Tecnológico de Roque, Km. 8. Carretera Celaya-J. Rosas. C.P. 38110. Tel. (01) 4616116362 ext. 142. Celaya, Guanajuato, México. frcervantes@itroque.edu.mx, covarrubiasjrg@hotmail.com, arangel_1@yahoo.com,mmendoza66@hotmail.com

3 Instituto Nacional de Investigaciones Forestales, Agrícolas y Pecuarias (INIFAP), Campo Experimental Bajío (CEBAJ). km. 6.5 carretera Celaya-San Miguel Allende. Celaya, Guanajuato, México. inifappreciado@prodigy.net.mx, inifapterron@prodigy.net.mx
} 


\section{INTRODUCCIÓN}

El maíz (Zea mays L.) es el cultivo más importante de México, desde el punto de vista alimentario, económico, político y social. Este grano se produce en dos ciclos agrícolas: primavera-verano y otoño-invierno, bajo diversas condiciones agroclimáticas de humedad: secano (temporal), punta de riego y riego (SIAP 2007).

Desde el punto de vista económico, el maíz se siembra en más de ocho millones de hectáreas, que representa $39 \%$ de la superficie agrícola nacional y $63 \%$ de la superficie sembrada con granos y oleaginosas; contribuye con $8 \%$ del producto interno de la agricultura y es el cultivo que más fuerza de trabajo ocupa. No obstante, se importaron ocho millones de toneladas de grano de maíz en 2006 y diez millones en 2010, lo que pone a este alimento a la cabeza de las importaciones de productos agrícolas de México.

En el año 2009 en México se sembraron aproximadamente ocho millones de hectáreas de maíz de las cuales se estima que 6,5 millones se sembraron con semilla nativa de polinización libre y 1,5 millones con semilla mejorada de calidad (SIAP 2010). Esta condición representa un bajo uso de semilla certificada (20\%) y bajo esta situación no se podrá lograr incrementar los rendimientos de este cultivo para llegar a ser autosuficientes. En este sentido, la FAO ha pronosticado que para el año 2050 la población Mundial superará los 9000 millones de personas y ha calculado que a fin de alimentar y mantener en buena salud a esa población la producción agrícola tendrá que crecer en un $70 \%$. Esta misma organización establece que disponer de suficientes semillas de calidad de variedades mejoradas es condición indispensable a fin de incrementar la producción alimentaria.

Los cambios que se producen en el rendimiento con el aumento de la densidad de plantas incrementan al principio y luego descienden y que estos cambios, en realidad son de la forma de una parábola, además que los bajos rendimientos a bajas densidades de siembra son debido a la escasez de plantas y en altas densidades de población provocan esterilidad (Ajamnouroozi y Bohrani 1998); también afirman, que la densidad de plantas en cada región está determinada por la competencia para la obtención de agua, luz y nutrientes.

La densidad de población ha sido un factor muy importante para contribuir a incrementar el rendimiento en maíz. Por tal motivo, varias investigaciones han estudiado el efecto de la densidad de población en la producción de semilla de maíz (Sprague 1985, Gutiérrez y Luna 2002) indicando que la densidad óptima de plantas para producción de semilla puede variar entre 40 y $100 \mathrm{mil}$ plantas/ha.

Al incrementar la densidad de población en maíz (33 300, 44400 y 66600 plantas/ha), se incrementó el rendimiento y el número de mazorcas por $\mathrm{m}^{2}$. Sin embargo, el número de semillas por mazorca fue mayor con 33300 planta/ha y el peso de grano disminuyó con el incremento en la densidad de población (Roy y Biswas 1992). De la Cruz et al. (2009) llegaron a las mismas conclusiones para rendimiento en poblaciones de maíz tropical. Por otro lado, Kresovic et al. (1997) concluyeron que un aumento en la densidad de 49300 a 59500 plantas/ha, el rendimiento de grano incrementó de 11,14 a 15,02 toneladas por hectárea en híbridos de maíz. Sin embargo, Nielson (1988) informó que con un aumento en la densidad de 44444 a 88888 plantas/ha, la producción de maíz aumentó en un 2,7 por ciento. Además señala que el estrés por agua y la falta de nitrógeno requerido por las plantas, también puede aplicar presión sobre el crecimiento y el rendimiento de maíz.

Con el incremento en la densidad de población disminuye el número de plantas que producen mazorca, aunque aumenta el rendimiento por unidad de área (González et al. 1984). Oyervides et al. (1990) evaluaron 40, 80 y $120 \mathrm{mil}$ plantas/ha en líneas de maíz, encontrando que los aumentos en la densidad de población indujeron una mayor altura de planta, retrasaron la floración masculina y femenina y hubo una reducción en el número de mazorcas por planta.

Se determinó la influencia de la densidad de población y la fertilización nitrogenada en el rendimiento de maíz en Nebraska, USA, y se concluyó que con un incremento en la población se aumenta el rendimiento (Blumenthal et al.2003). Por otro lado, este respondió favorablemente al incremento en la fertilización nitrogenada (Bravo y Chan 1987).

En evaluaciones del efecto de la fertilización nitrogenada, se menciona que en maíz el peso del grano y el número por mazorca están influenciados positivamente por la disponibilidad de nitrógeno (Lemcoff y Loomis 1986). Evaluaciones de una cruza simple de maíz en dos dosis de nitrógeno (0 y $167 \mathrm{~kg} / \mathrm{ha}$ ) y dos densidades de población (36 600 y 73200 plantas/ 
ha), muestran que el rendimiento de grano estuvo más relacionado con la densidad de población que con la fertilización nitrogenada, y fue más dependiente del número de granos por mazorca que del tamaño de los mismos; en este sentido, hubo una reducción del peso de grano y mazorca con el incremento en la densidad de población (Lemcoff y Loomis 1994). Por su parte, Stamp et al. (2000), al comparar dos dosis de N (0 y $200 \mathrm{~kg} / \mathrm{ha}$ ) en híbridos de maíz, encontraron que el estrés ocasionado por la deficiencia de nitrógeno redujo el número de granos por mazorca. Tanaka y Yamaguchi (1981) llegaron a las mismas conclusiones para el índice de prolificidad con niveles bajos de nitrógeno. En este sentido, Barbieri et al. (2000) concluyeron que con bajos niveles de nitrógeno disminuyó el número de granos por mazorca y el rendimiento; mientras que al reducir la distancia entre surcos estos caracteres se incrementaron significativamente.

Evaluaciones de tres dosis de fertilización y cuatro densidades de población $(45,60,75$ y 80 mil plantas/ha) en dos cruzas simples de maíz, muestran que es recomendable utilizar la densidad de $60 \mathrm{mil}$ plantas/ha en los lotes de producción de semilla donde las cruzas simples se utilicen como progenitores hembra, ya que, además de tener un buen rendimiento de semilla, se facilita el manejo del cultivo. También se señala que la respuesta típica de las plantas a suelos de baja fertilidad conduce a la reducción de la cantidad de semilla producida, más que en su calidad (Espinosa y Tadeo 1992). Muchos científicos creen que una mala aplicación del riego y la escasez del nitrógeno requerido por la planta son los factores más importantes para disminuir el rendimiento del maíz. En muchos estudios, se afirma un efecto positivo del nitrógeno en el rendimiento de grano, en el número de semillas por mazorca y en el peso de la semilla de híbridos de maíz (Osborne et al. 2002). En un estudio para evaluar la respuesta de cruzas simples de maíz a diferentes densidades de población y niveles de nitrógeno, Yasari et al. (2012), encontraron que niveles altos de nitrógeno $(350 \mathrm{~kg} / \mathrm{ha})$ incrementaron el rendimiento de grano. Por otro lado, altas densidades de población (95000 plantas/ha) disminuyen el número de semillas por hilera y de hileras por mazorca; mientras que la aplicación del nitrógeno no afectó estos caracteres.

Hay un efecto negativo del fósforo, solo o interactuando con el nitrógeno o potasio en la germinación (Jocely 1979). Este autor concluyó que las pruebas de vigor no revelaron influencia alguna de la fertilización en la calidad de la semilla. Martínez et al. (2005) al evaluar densidades de población y dosis de fertilización en la producción de semilla híbrida de maíz, obtuvieron que estos factores no mostraron efecto significativo en la calidad de la semilla a través de la prueba de germinación estándar. La calidad fisiológica de la semilla de maíz también ha sido estudiada a nivel genético, para vigor de esta en líneas endogámicas de maíz tropical (Cervantes et al. 2006, Cervantes et al. 2011) y para vigor de semilla en líneas endogámicas de maíz tropical.

De acuerdo con lo anterior, se pudo apreciar que la producción de semilla híbrida de maíz requiere de productores especializados, ya que las condiciones para lograrlo deben ser óptimas debido a la diversidad de factores que afectan el rendimiento y calidad de la semilla. Por lo anterior y con la finalidad de contribuir a resolver parte de la problemática en la producción de semilla de maíz en México, este estudio tuvo como objetivo determinar el efecto de la densidad de población y la fertilización nitrogenada en la producción y calidad física y fisiológica de semilla de maíz en el Bajío Guanajuatense.

\section{MATERIALES Y MÉTODOS}

El estudio se realizó en dos fases: la primera en campo y la segunda en laboratorio. En la investigación de campo se realizaron dos experimentos durante el ciclo Primavera-Verano 2009 en los terrenos del Campo Experimental Bajío (CEBAJ) del Instituto Nacional de Investigaciones Forestales, Agrícolas y Pecuarias (INIFAP), ubicado en Celaya, Guanajuato, México $\left(20^{\circ} 31^{\prime}\right.$ ' Latitud norte, $100^{\circ} 45^{\prime}$ ' longitud oeste y 1765 msnm). El clima del sitio experimental es semicálido $\mathrm{BS} 1 \mathrm{Hw}(\mathrm{e})$, con una precipitación de 550 a $710 \mathrm{~mm}$ durante el año y temperatura media anual de $18,4^{\circ} \mathrm{C}$ (García 1973). Los suelos son de tipo Vertisol Pélico, se caracterizan por ser arcillosos de coloración oscura.

En el primer experimento, el material genético utilizado fue el progenitor femenino de cruza simple (CML 176 x CML 142), y la línea endogámica CML 186, progenitores del híbrido trilineal H-374C. En el segundo experimento, se formó el híbrido de cruza simple de alta calidad de proteína H-469C, con una línea endogámica como progenitor femenino (CML 176) y el mismo progenitor masculino del 
experimento anterior. Los materiales genéticos para ambos híbridos (H-374C y H-469C) fueron derivados por el Centro Internacional de Mejoramiento de Maíz y Trigo (CIMMYT 1998) los cuales se describen a continuación: CML 176 es una línea endogámica de tres autofecundaciones cuya genealogía (P63-12-2-1/ P67-5-1-1)-1-2-B-B) es derivada de un cruzamiento de líneas S3 de las poblaciones 63 (de origen tropical, blanco dentado 1 QPM) y 67 (de origen subtropical, templado blanco cristalino-QPM). CML 142 es una línea endogámica de cinco autofecundaciones cuya genealogía (P62C5HC93-5-6-1-3-B-B-B-7- B-B-\#) es derivada de la población 62 (de origen tropical y subtropical). La CML 186 es una línea endogámica de tres autofecundaciones cuya genealogía es (P67C2HC26-1-2-1-B-B), derivada de la población 67 templado blanco cristalino-QPM (origen subtropical).

Se evaluaron dos factores, densidad de población con los niveles 60,75 y 90 mil plantas/ha y fertilización nitrogenada con los niveles 150,250 y $350 \mathrm{~kg}$ de N/ha para la cruza simple CML 176 x CML 142 progenitor femenino del híbrido de cruza triple H-374C y para la línea CML 176 progenitor femenino del híbrido de cruza simple H-469C. Se estudiaron los mismos factores del experimento anterior; manteniéndose los niveles de nitrógeno y variando las densidades de población de 60, 80 y $100 \mathrm{mil}$ plantas/ha.

Para los dos experimentos el progenitor femenino se sembró en seco el 14 de junio y el masculino el 21 de junio de 2009. Debido a que el trabajo se realizó simulando lotes de producción de semilla con una relación 6:2, cada unidad experimental estuvo constituida por seis surcos para los progenitores femeninos y dos surcos para los masculinos; con $6 \mathrm{~m}$ de longitud y una separación de $0,76 \mathrm{~m}$. Todos los surcos hembra fueron desespigados de forma manual para permitir únicamente la fecundación con el macho polinizador. Además el arreglo en la orientación de los surcos fue la adecuada; ya que el experimento estuvo ubicado con la orientación norte-sur y la predominancia de los vientos tienen la dirección este-oeste; lo cual facilitó la polinización natural completa de todos los surcos hembra, y si a esto se le suma la capacidad y el intervalo de producción de polen (8-10 días) del macho, se puede indicar que el progenitor femenino fue polinizado completamente.

En ambos experimentos se usó un diseño experimental de parcelas divididas con nueve tratamientos y cuatro repeticiones; a la parcela principal se le asignó la fertilización nitrogenada y a la pequeña la densidad de población.

En los dos experimentos de campo, al momento de la siembra se aplicó en banda el 50\% del nitrógeno, todo el fósforo y el potasio, estos últimos en dosis de $60 \mathrm{~kg} / \mathrm{ha}$; la otra mitad del nitrógeno se aplicó 40 días después de la siembra (dds). Se sembró una densidad mayor a la requerida en los experimentos y a los 20 dds se ajustó a la población deseada.

Se aplicaron tres riegos de auxilio por la buena distribución de las lluvias. Para mantener el cultivo libre de malezas se aplicó el herbicida atrazina (25\%) y metolachlor (25\%) en dosis de 4 1/ha y se hicieron dos escardas mecánicas (20 y 40 dds). Para el control de plagas del suelo se aplicó el insecticida granulado carbofuran, mezclado con el fertilizante al momento de la siembra.

En los dos experimentos se determinó: altura de planta (AP) y de mazorca (AM), días a floración femenina (DFF), índice de prolificidad (IP) y rendimiento de semilla en campo (RSC). La altura de planta y mazorca se determinó tomando tres plantas al azar de los surcos centrales; el rendimiento de semilla en campo se obtuvo de cosechar los dos surcos centrales, eliminando una planta en cada extremo. La floración femenina se determinó en el progenitor femenino, considerando los días transcurridos entre el primer riego y la fecha en que el $50+1 \%$ de las plantas de cada unidad experimental mostraron los estigmas entre 2 y $3 \mathrm{~cm}$ de largo. El índice de prolificidad se determinó al dividir el número de mazorcas cosechadas entre el número de plantas de la parcela útil. Por otra parte, en diez mazorcas tomadas al azar de cada unidad experimental se determinaron los siguientes caracteres: diámetro de mazorca (DM), longitud de mazorca (LM) y número de semillas por hilera (NSH). En semilla se determinó el peso volumétrico y el peso de 1000 semillas de acuerdo a la metodología reportada por la ISTA (1999), y la calidad fisiológica con una prueba de vigor, esta a través de la prueba de envejecimiento acelerado (EA), seguida por la prueba de germinación estándar (GE) (Moreno 1996). También se hizo una clasificación por forma (planos y bolas) y tamaño (grande, mediano y pequeño) de la semilla, utilizando diferentes tipos de cribas (forma y tamaños); estos últimos caracteres 
de semilla, se llevaron a cabo en el Laboratorio de Análisis de Semillas del Instituto Tecnológico de Roque (I.T. Roque), Celaya, Gto.

Las variables de laboratorio, determinadas en porcentajes, se transformaron ( $\arcsin )$ para su análisis, usando el procedimiento PROC ANOVA del paquete estadístico SAS (SAS 1999) ver. 8.1. La comparación múltiple de medias se realizó de acuerdo a la prueba de Tukey $(\mathrm{P} \leq 0,05)$.

\section{RESULTADOS Y DISCUSIÓN}

Cruza CML 176 x CML 142. En los resultados del análisis de varianza (ANOVA) de las características agronómicas, la densidad de población mostró diferencias significativas $(\mathrm{P} \leq 0,01)$ en altura de mazorca, días a floración femenina, rendimiento de semilla en campo y para el índice de prolificidad. La fertilización nitrogenada no tuvo efecto significativo en este grupo de caracteres; la interacción de los factores sólo afectó estadísticamente al rendimiento de semilla en campo. Gutiérrez y Luna (2002) obtuvieron resultados similares en relación con el efecto del nitrógeno. Pero difieren de los resultados encontrados por Tanaka y Yamaguchi (1981), Bravo y Chan (1987), Lemcoff y Loomis (1986) y Blumenthal et al. (2003) quienes reportaron que el rendimiento responde favorablemente a la aplicación de nitrógeno. Por otro lado, estos resultados confirman los publicados por Yasari (2012) en relación a la interacción de los factores para rendimiento de semilla en campo.

El incremento de la densidad de población retrasó ligeramente los días a floración femenina, debido a la competencia entre plantas (Cuadro 1). Estos resultados coinciden con los de Oyervides et al. (1990) y se contraponen a los de Espinosa y Tadeo (1992) quienes no encontraron diferencias en floración al incrementar la densidad de población. La altura de mazorca fue mayor con el incremento de 60 a 75 mil plantas/ha, y se mantuvo con el incremento a 90 mil plantas/ha. Lo anterior puede ser debido al efecto de la competencia y menor disponibilidad de nutrimentos.

El rendimiento de semilla de campo mostró una tendencia positiva al incrementar la densidad de población. La densidad de población media y alta (75 y $90 \mathrm{mil} \mathrm{plantas/ha)} \mathrm{fueron} \mathrm{superiores} \mathrm{estadísticamente}$ en el rendimiento de semilla que la densidad baja (60 mil plantas/ha), debido al mayor número de plantas, a pesar de no haber diferencias estadísticas en los caracteres de mazorca; este incremento pudiera deberse al mayor número de ellas. Este resultado es probable, ya que aunque se espera una reducción en el tamaño de mazorca, este se compensa por el mayor número de ellas como lo indican Feizbakhsh et al. (2007). Resultados similares fueron reportados por Sprague (1985),

Cuadro 1. Comparación de medias para caracteres agronómicos del híbrido de maíz de cruza simple (CML 176 x CML 142), progenitor femenino del H-374C. CEBAJ, INIFAP. Celaya, Guanajuato, México. 2009.

\begin{tabular}{lcccc}
\hline $\begin{array}{l}\text { Factores y niveles } \\
\text { de estudio }\end{array}$ & $\begin{array}{c}\text { AM } \\
(\mathbf{c m})\end{array}$ & $\begin{array}{c}\text { DFF } \\
(\text { días })\end{array}$ & $\begin{array}{c}\text { RSC } \\
\text { (kg/ha) }\end{array}$ & $\begin{array}{c}\text { IP } \\
(\mathbf{n o} .)\end{array}$ \\
\hline Densidad de población (plantas/ha) & & & $1,25 \mathrm{a}$ \\
\hline 60000 & $167,9 \mathrm{~b}$ & $76 \mathrm{~b}$ & $6868,3 \mathrm{~b}$ & $1,19 \mathrm{a}$ \\
75000 & $177,1 \mathrm{ab}$ & $77 \mathrm{ab}$ & $7276,5 \mathrm{ab}$ & $1,04 \mathrm{~b}$ \\
90000 & $177,2 \mathrm{a}$ & $78 \mathrm{a}$ & $7772,8 \mathrm{a}$ & $1,19 \mathrm{a}$ \\
\hline Dosis de nitrógeno (kg/ha) & & & $7391,4 \mathrm{a}$ & $1,17 \mathrm{a}$ \\
\hline 150 & $168,6 \mathrm{a}$ & $77 \mathrm{a}$ & $7304,8 \mathrm{a}$ & $1,10 \mathrm{a}$ \\
\hline
\end{tabular}

AM: altura de mazorca, DFF: días a floración femenina, RSC: rendimiento de semilla en campo, IP: índice de prolificidad. Medias con letras iguales en cada columna no son estadísticamente diferentes (Tukey, 0,05). 
Esechie (1992), Gutiérrez y Luna (2002), Blumenthal et al. (2003) y Yasari (2012), quienes encontraron que usualmente el rendimiento de semilla se incrementa significativamente con el incremento en la densidad de población. Sin embargo, se difieren de los resultados de Espinosa y Tadeo (1992) ya que ellos recomiendan $60 \mathrm{mil}$ plantas/ha para la producción de semilla de cruza simple. El incremento de 75 a 90 mil plantas/ ha no afecta de forma significativa el rendimiento de semilla; lo cual indica, que la hembra de cruza simple (CML 176 x CML 142) del híbrido H-374C no tolera altas densidades de siembra por lo que debe establecerse a una densidad de 75 mil plantas/ha para lotes de producción de semilla de alta calidad.

El índice de prolificidad (número de mazorcas por planta) disminuyó con el incremento en la densidad de población de 1,25 (60 mil plantas/ha) a 1,04 con 90 mil plantas/ha. Esto se debe a que en niveles altos de población incrementa el número de plantas "horras" o estériles por efecto de competencia (González et al. 1984, Oyervides et al. 1990, Roy y Biswas 1992).

Las variables de característica de mazorca no presentaron efectos estadísticos significativos tanto para la densidad de población como para la fertilización nitrogenada. Esto demuestra que estas variables pueden ser mayormente influenciadas por el genotipo o por el ambiente. Estos resultados no coinciden con los encontrados por Oyervides et al. (1990) y Roy y Biswas (1992) al concluir que existe efecto negativo con el incremento de la densidad de población en los componentes de rendimiento. Por otro lado, Lemcoff y Loomis (1986), Lemcoff y Loomis (1994) y Osborne (2002) concluyeron que la disponibilidad de nitrógeno tiene efecto positivo en el peso de grano y el número de ellos por mazorca. De la misma manera, Barbieri et al. (2000) y Stamp et al. (2000) señalan que el estrés de nitrógeno disminuye el número de granos por mazorca y el rendimiento total.

Los resultados del ANOVA para las variables de calidad física y fisiológica de la semilla, muestran que la densidad de población y la fertilización nitrogenada no tuvieron efectos estadísticos significativos en ninguno de los caracteres evaluados. Estos resultados concuerdan con los de Jocely (1979), Gutiérrez y Luna (2002) y Martínez et al. (2005) al evaluar el vigor semilla. Pero difieren de los resultados de Roy y Biswas (1992) y Oyervides et al. (1990) al indicar que con densidades altas, el peso de semilla disminuye significativamente. Espinosa y Tadeo (1992) señalan que la respuesta típica de las plantas a suelos de baja fertilidad conduce a la reducción de la cantidad de semilla producida más que en su calidad. La interacción nitrógeno por densidad de población sólo mostró efecto significativo $(\mathrm{P} \leq 0,05)$ para el peso volumétrico (PV) y para vigor de semilla a través de la prueba de envejecimiento acelerado (EA). Lo anterior demuestra que estos factores individuales con los niveles evaluados no afectan estas características de calidad de la semilla.

El análisis de varianza para los caracteres de clasificación de semilla (forma y tamaño), presenta ausencia de efectos estadísticos para este grupo de caracteres; lo que pudiera atribuirse a que el tamaño de muestra no fue el adecuado $(4 \mathrm{~kg})$ para representar las diferentes clases de semillas o la maquinaria utilizada para la clasificación; ya que esta se utiliza para lotes semicomerciales de semilla y a esto se le atribuye la alta variación en este grupo de caracteres.

Línea CML 142. En los resultados de análisis de varianza muestran que la densidad de población presentó efecto significativo en altura de mazorca $(\mathrm{P} \leq 0,05)$, días a floración femenina $(\mathrm{P} \leq 0,01)$, rendimiento de semilla en campo $(P \leq 0,05)$ y el índice de prolificidad $(\mathrm{P} \leq 0,01)$. La fertilización nitrogenada únicamente afectó estadísticamente el índice de prolificidad (P). No hubo interacción significativa de estos factores en este grupo de caracteres evaluados.

Con respecto a la aplicación de nitrógeno, se ha señalado que el rendimiento responde favorablemente con el incremento en la fertilización nitrogenada (Bravo y Chan 1987, Barbieri et al. 2000, Osborne 2002, Blumental et al. 2003).

En los resultados de la prueba de comparación de medias (Cuadro 2), se observa que existió un retraso en la floración de un día con 100000 plantas/ha en relación a 60000 y 80000 plantas/ha. La altura de mazorca se incrementó cuando la población pasó de 60000 a 80000 plantas/ha; pero esta disminuyó con 100000 plantas/ha. Estos resultados están de acuerdo con los reportados por Oyervides et al. (1990) para floración. Con relación al rendimiento de semilla en campo, se puede observar que la densidad de población de 60000 plantas/ha presentó el rendimiento más bajo pero se incrementó con la población de 80 000 plantas/ha. Resultados similares han sido obtenidos por varios autores (Sprague 1985, Nielson 1988, 
Cuadro 2. Comparación de medias para variables agronómicas y de características de mazorca en la línea CML 176 progenitor femenino del híbrido H-469C. CEBAJ, INIFAP. Celaya, Guanajuato, México. 2009.

\begin{tabular}{|c|c|c|c|c|c|c|c|}
\hline $\begin{array}{l}\text { Factores y niveles } \\
\text { de estudio }\end{array}$ & $\begin{array}{c}\mathrm{AM} \\
(\mathbf{c m})\end{array}$ & $\begin{array}{l}\text { DFF } \\
\text { (días) }\end{array}$ & $\begin{array}{c}\text { IP } \\
\text { (no.) }\end{array}$ & $\begin{array}{c}\text { RSC } \\
\text { (kg/ha) }\end{array}$ & $\begin{array}{l}\mathbf{L M} \\
(\mathrm{cm})\end{array}$ & $\begin{array}{l}\text { DM } \\
(\mathbf{c m})\end{array}$ & $\begin{array}{l}\text { NSH } \\
\text { (no.) }\end{array}$ \\
\hline \multicolumn{8}{|c|}{ Densidad de población (plantas/ha) } \\
\hline 60000 & $99,9 \mathrm{a}$ & $82 \mathrm{~b}$ & $0,83 \mathrm{a}$ & $2492,2 \mathrm{~b}$ & $11,33 \mathrm{a}$ & $3,14 \mathrm{ab}$ & $18,31 \mathrm{a}$ \\
\hline 80000 & $105,9 a b$ & $82 \mathrm{~b}$ & $0,81 \mathrm{a}$ & $2869,3 \mathrm{ab}$ & $10,94 \mathrm{~b}$ & $3,25 \mathrm{a}$ & $17,51 \mathrm{ab}$ \\
\hline 100000 & 104,6 b & $83 \mathrm{a}$ & $0,70 \mathrm{~b}$ & $2924,2 \mathrm{a}$ & $10,36 \mathrm{c}$ & $3,02 \mathrm{~b}$ & $16,92 \mathrm{~b}$ \\
\hline \multicolumn{8}{|c|}{ Dosis de nitrógeno (kg/ha) } \\
\hline 150 & $104,0 \mathrm{a}$ & $82 \mathrm{a}$ & $0,80 \mathrm{ab}$ & $2874,4 \mathrm{a}$ & $11,15 \mathrm{a}$ & $3,26 \mathrm{a}$ & $17,80 \mathrm{a}$ \\
\hline 250 & $104,4 \mathrm{a}$ & $82 \mathrm{a}$ & $0,82 \mathrm{a}$ & $2798,6 \mathrm{a}$ & $11,01 \mathrm{a}$ & $3,17 \mathrm{ab}$ & $17,65 \mathrm{a}$ \\
\hline 350 & $104,0 \mathrm{a}$ & $82 \mathrm{a}$ & $0,72 \mathrm{~b}$ & $2612,4 \mathrm{a}$ & $10,47 \mathrm{~b}$ & $2,98 \mathrm{~b}$ & $17,30 \mathrm{a}$ \\
\hline
\end{tabular}

AM: altura de mazorca, DFF: días a floración femenina, IP: índice de prolificidad, RSC: rendimiento de semilla de campo, LM: longitud de mazorca, DM: diámetro de mazorca, NSH: número de semillas por hilera.

Medias con letras iguales en cada columna no son estadísticamente diferentes (Tukey, 0,05).

Oyervides et al. 1990, Roy y Biswas 1992, Espinosa y Tadeo 1992, Kresovic et al. 1997, Gutiérrez y Luna 2002, De la Cruz et al. 2009, Yasari et al. 2012). El índice de prolificidad disminuyó con el incremento en la densidad de población (100 mil plantas/ha) así como también con la fertilización nitrogenada de $350 \mathrm{~kg}$ de N/ha; comportamiento esperado ya que las altas densidades provocan mayor competencia y, por consiguiente, un mayor número de plantas "horras" o estériles; con respecto al efecto del nitrógeno en las líneas el nivel de endogamia pudiera impedir que respondan a este factor, por lo que necesitan niveles de nitrógeno moderados o bajos. Con aplicaciones de 150 y $250 \mathrm{~kg}$ de N/ha se obtuvo el mayor índice de prolificidad $(0,80$ y 0,82 , respectivamente). Estos resultados están de acuerdo con los de Oyervides et al. (1990) con respecto al efecto de la densidad de plantas, pero no coinciden con los reportados por Lemcoff y Lomis (1994), Tanaka y Yamaguchi (1981) y Stamp et al. (2000) quienes señalan que con niveles bajos de nitrógeno no se expresa la prolificidad.

Con respecto a los caracteres de mazorca en la línea CML 176, el factor densidad de población presentó significancia estadística para las variables longitud y diámetro de mazorca ( $\mathrm{p} \leq 0,01$ y $\mathrm{p} \leq 0,05$, respectivamente) y número de semillas por hilera $(\mathrm{P} \leq 0,05)$. La fertilización nitrogenada tuvo efectos estadísticos $(\mathrm{p} \leq 0,01)$ en la longitud y diámetro de mazorca. La interacción de estos factores no mostró efecto significativo para este grupo de variables. Estos resultados difieren de lo reportado por Yasari (2012) quien señala una interacción significativa en estos factores de estudio.

Los resultados de la prueba de comparación de medias, indican que la mayor longitud de mazorca se asoció con la densidad de 60000 plantas/ha y este disminuyó al incrementar la densidad a 80000 y 100 000 plantas/ha, mientras que el mayor diámetro de mazorca se asoció con 80000 plantas/ha aunque no hubo diferencias significativas con 60000 plantas/ha (Cuadro 2), comportamiento esperado ya que las altas densidades provocan competencia entre plantas, y por lo tanto se presenta una respuesta negativa en el diámetro y longitud de mazorca (Oyervides et al. 1990, Lemcoff y Loomis 1994, Feizbakhsh et al. (2007). El número de semillas por hilera (NSH) disminuyó significativamente con el incremento en la densidad de población, este hecho se atribuye que a altas densidades se forman mazorcas pequeñas y con ello menor número de semillas por hilera (Roy y Biswas 1992, Feizbakhsh et al.2007). El incremento de la fertilización nitrogenada tuvo efectos negativos en el diámetro y longitud de mazorca. En este tópico, Lemcoff y Loomis (1986), 
Barbieri et al. (2000) y Stamp et al. (2000) indican que la aplicación de nitrógeno modifica positivamente algunas características de componente de rendimiento. Por otro lado, Lemcoff y Loomis (1994) señalan que el rendimiento tiene mayor relación con la densidad de población que con la fertilización nitrogenada.

En el análisis de varianza, para las variables de calidad física y fisiológica de la semilla, los cambios en los niveles de población y la aplicación de nitrógeno no mostraron presencia de efectos significativos en estos caracteres. Estos resultados están de acuerdo con Jocely (1979), Espinosa y Tadeo (1992) y Martínez et al. (2005) quienes encontraron que la fertilización nitrogenada y la densidad de plantas no tienen efecto en la calidad de la semilla. Sin embargo, Oyervides et al. (1990) y Lemcoff y Loomis (1994) encontraron una reducción en el peso de semilla con el incremento en la densidad de población en líneas de maíz.

Por otro lado, en los caracteres de clasificación de semilla la densidad de población sólo presentó diferencias significativas $(\mathrm{P} \leq 0,05)$ en las clases desecho de semilla, semilla bola grande y en el porcentaje de semilla útil $(\mathrm{P} \leq 0,01)$. Por su parte, los cambios en los niveles de nitrógeno no mostraron efectos estadísticos significativos para este grupo de variables).

La prueba de comparación de medias indica que el porcentaje de desecho de semilla se incrementó con la densidad alta (100 000 plantas/ha), mientras que el porcentaje de semilla bola grande y de semilla útil disminuyeron (Cuadro 3), este comportamiento era esperado debido a que a altas densidades se producen mazorcas y granos más pequeños, por lo que se presenta mayor porcentaje de deshecho de semilla y por lo tanto, disminuye el porcentaje de semilla útil.

En términos generales se puede establecer que la densidad de población óptima estaría en el rango de 75 a 90 mil plantas/ha para la cruza simple CML 142 x CML 176, progenitor femenino del híbrido trilineal QPM H-374C, por ser consistente con el mayor rendimiento de campo. En la línea de maíz CML-176, progenitor femenino del híbrido de cruza simple QPM H-469C, se obtiene que la densidad de 80 mil plantas/ha produce el mayor rendimiento de semilla de campo.

Por otro lado, la línea CML 176, con 100 mil plantas provocó efecto negativo en la longitud y el diámetro de mazorca, el número de semillas por hilera, el porcentaje de bola grande y porcentaje de semilla útil.

Por último, se considera que las dosis de 250 y $350 \mathrm{~kg} \mathrm{~N} / \mathrm{ha}$ no son recomendables en la producción de semilla híbrida de maíz de los materiales evaluados, por no mejorar el rendimiento y la calidad física y fisiológica de semilla.

Cuadro 3. Comparación de medias para los caracteres de clasificación de semilla en la línea CML 176 progenitor femenino del híbrido H-469C. I.T Roque. Celaya, Guanajuato, México. 2009.

\begin{tabular}{llll}
\hline Factores y niveles de estudio & DS & BG & SU \\
\cline { 2 - 4 } & & $(\%)$ & $77,8 \mathrm{a}$ \\
\hline Densidad de población (plantas/ha) & & & $77,9 \mathrm{a}$ \\
\hline 60000 & $12,1 \mathrm{~b}$ & $20,5 \mathrm{a}$ & $74,6 \mathrm{~b}$ \\
80000 & $11,9 \mathrm{~b}$ & $20,0 \mathrm{ab}$ & $77,9 \mathrm{a}$ \\
100000 & $14,5 \mathrm{a}$ & $16,4 \mathrm{~b}$ & $76,9 \mathrm{a}$ \\
\hline Dosis de nitrógeno (kg/ha) & & & $75,6 \mathrm{a}$ \\
\hline 150 & $12,1 \mathrm{a}$ & $19,5 \mathrm{a}$ & $18,9 \mathrm{a}$ \\
\hline 50 & $12,9 \mathrm{a}$ & $15,5 \mathrm{a}$ & \\
\hline
\end{tabular}

Medias con letras iguales en cada columna no son estadísticamente diferentes (Tukey 0,05).

DS: desecho de semilla, BG: bola grande, SU: semilla útil. 


\section{LITERATURA CITADA}

Ajamnouroozi, H; Bohrani, J. 1998. The effects of planting configuration and plant density on the yield and yield components of the late maturing grain corn cultivar of S. C 704 and the intermediate maturing corn cultivar of S. C 704 in the region of Aliabad Kamin in the province of Fars. In Proceeding $5^{\text {th }}$ Congress of Iranian Agronomy and Plant Breeding. Karaj, Iran. p. 380.

Barbieri, P; Sainz, H; Andrade, F; Echeverria, H. 2000. Row spacing effects at different levels of nitrogen availability in maize. Agronomy Journal 92:283-288.

Blumenthal, J; Lyon, D; Stroup, W. 2003. Optimal plant population and nitrogen fertility for dryland corn in western Nebraska. Agronomy Journal 95:878-883.

Bravo, A; Chan, J. 1987. Relaciones agua-suelo-plantaatmósfera del maíz de riego en zonas semiáridas. I. Rendimiento de grano. Terra 5(2):132-139.

Cervantes, F; García, G; Carballo, A; Bergvinson, D; Crossa, J; Mendoza, M; Moreno, E. 2006. Análisis dialélico para caracteres de vigor de semilla y de plántula en genotipos de maíz tropical. Agricultura Técnica en México 32(1):77-87.

Cervantes, F; García, G; Carballo, A; Bergvinson, D; Crossa, J; Mendoza, M; Andrio, E; Rivera, G; Moreno, E. 2011. Estimación de efectos genéticos relacionados con el vigor de la semilla y de la plántula en maíces tropicales mexicanos. International Journal of Experimental Botany 80:19-26.

CIMMYT (Centro Internacional de Mejoramiento de Maíz y Trigo). 1998. A complete listing of improved maize germoplasm from CIMMYT. Maize Program Special Report. México, D.F. 94 p.

De la Cruz, E; Cordoba, H; Estrada, M; Mendoza, J; Gómez, A; Brito, N. 2009. Rendimiento de grano de genotipos de maíz sembrados bajo tres densidades de población. Universidad y Ciencia 25(1):93-98.

Esechie, H. 1992. Effect of planting density on growth and yield of irrigated maize (Zea mays) in the Batinah Coast region of Oman. Journal of Agricultural Science 119:165-169.

Espinosa, A; Tadeo, M. 1992. Producción de semilla del híbrido doble de maíz en respuesta a la fertilización nitrogenada y densidad de población. Rev. Fitotec. Mex. 15:1-9.

FAO (Food and Agriculture Organization). 2010. Invertir en seguridad alimentaria. Organización de las Naciones Unidas para la Agricultura y Alimentación (FAO). Viale delle Terme di Caracalla 00153. Roma, Italia. 35 p.
Feizbakhsh, M; Nemati, H; Mokhtarpour, A; Mossavat, A; Saberi, F; Sheikh, F. 2007. The effect of eliminating shoots and that of plant density on the yield and its components of sweet corn. Journal of Constructive Research in Agronomy and Horticulture 77:66-69.

García, E. 1973. Modificaciones al sistema de clasificación climática de Koppen (adaptación a las condiciones de la República Mexicana). Instituto de Geografía de UNAM, D.F. México. 264 p.

González, V; Ortiz, J; Mendoza L. 1984. Rendimiento del maíz y sus componentes en respuesta a diversas prácticas culturales y criterios de selección. Agrociencia 58:101-112.

Gutiérrez, R; Luna, M. 2002. Riego, densidad de plantas y fertilización nitrogenada en producción de híbridos de maíz en Zacatecas. Agricultura Técnica en México 28(2):95-103.

ISTA (International Seed Testing Association). 1999. International rules for seed testing. Zurich, Switzerland. $321 \mathrm{p}$.

Jocely, A. 1979. Influencia de adubacao mineral NPK sobre a qualidade de semente de milho. Bragantia 38:165174.

Kresovic, B; Tolimir, M; Pajic, Z. 1997. Growing of sweet corn as a second or stubble crop. J. of Sci. Agric. Aes. 48:23-30.

Lemcoff, J; Loomis, R. 1986. Nitrogen influences on yield determination in maize. Crop Sci. 26:1017-1022.

Lemcoff, J; Loomis, R. 1994. Nitrogen and density influence on silk emergence, endosperm development and grain yield in maize (Zea mays L). Field Crop Research 38: 63-72.

Martínez, C; Mendoza, L; García, G; Martínez, A. 2005. Producción de semilla híbrida de maíz con líneas androfértiles y androestériles isogénicas y su respuesta a la fertilización y densidad de población. Rev. Fitot. Mex. 28(2):127-133.

Moreno, E. 1996. Análisis físico y biológico de semillas agrícolas. 3 ed. Instituto de Biología. UNAM. México. $393 \mathrm{p}$.

Nielson, RL. 1988. Influence of hybrids and plant density on grain yield and stair breakage in corn growth in 15inch row spacing. J. Prod. Agric. 190-195.

Osborne, L; Scheppers, S; Francis, D; Schlemmer, R. 2002. Use of spectral radiance to in-season biomass and grain yield in nitrogen and water-stressed corn. Crop. Sci. 42:165-171.

Oyervides, A; Ortiz, J; González, V; Carballo, A. 1990. El número de mazorcas por planta y la formación de arquetipos de maíz. Serie fitotecnia 1(4):103-118. 
Roy, S; Biswas, P. 1992 Effect of density and detopping following silking on cob growth, fodder and grain yield of maize (Zea mays). Journal of Agricultural Science 119:297-301.

SAS Institute Inc. 1999. SAS/STAT user's guide. Version 8.1 Cary, NC, USA. 359 p.

SIAP (Servicio de Información Agroalimentaria y Pesquera). 2007. Situación actual y perspectivas del maíz en México 1996 - 2012. Secretaría de Agricultura, Ganadería, Desarrollo Rural, Pesca y Alimentación (SAGARPA). México, D.F. 208 p.

SIAP (Servicio de Información Agroalimentaria y Pesquera). 2010. Estadísticas de producción de semilla del año agrícola 2009 (en línea). Consultado julio 2011. Disponible en http://www.siap.gob.mx/aagricola_siap/ icultivo/index.jsp
Sprague, G. 1985. Corn and corn improvement. Am. Soc. Agron. Inc. Publisher. Madison, Wisconsin, USA. p 645-648.

Stamp, P; Schowchong, S; Menzi, M; Weingarther, U; Kaiser, O. 2000. Increase in the yield of cytoplasmic male sterile maize revisited. Crop. Sci. 40:15861587.

Tanaka, A; Yamaguchi, J. 1981. Producción de materia seca, componentes de rendimiento y rendimiento de grano en maíz. $2^{a}$ impresión. Trad. por J. Kohashi, S. Rama de Botánica, Colegio de Postgraduados. Chapingo, Edo. de México. México. 124 p.

Yasari, E; Noori, M; Haddadi, M. 2012. Comparison of seed corn single coesses SC 704 and SC 770 response to different plant densities and nitrogen levels. Journal of Agricultural Science 4(5):263-272. 